\title{
Discrepancies in assessing anterior chamber activity among uveitis specialists
}

Running Title: Anterior chamber activity discrepancies

Tun Hang Yeo, ${ }_{1}^{1}$ Siddharthan Ilangovan, ${ }^{2}$ Pearse A Keane, ${ }^{3}$ Carlos Pavesio, ${ }^{4}$ Rupesh

$$
\text { Agrawal }^{1,4}
$$

${ }^{1}$ National Healthcare Group Eye Institute, Tan Tock Seng Hospital, Singapore

${ }^{2}$ Raffles Institution, Singapore

${ }^{3}$ NIHR Biomedical Research Centre for Ophthalmology, Moorfields Eye Hospital NHS Foundation Trust and UCL Institute of Ophthalmology, London, UK

${ }^{4}$ Moorfields Eye Hospital, NHS Foundation Trust, London, UK

\section{Corresponding author:}

Dr Tun Hang Yeo, National Healthcare Group Eye Institute, Tan Tock Seng Hospital, Singapore 308433

Email: tunhang.yeo@gmail.com

Declaration of interest: The authors have no conflicts of interest to report. The authors alone are responsible for the content and writing of the paper.

*Dr Keane received a proportion of his funding from the Department of Health's NIHR Biomedical Research Centre for Ophthalmology at Moorfields Eye Hospital 
and the UCL Institute of Ophthalmology. The views expressed in the publication are those of the author and not necessarily those of the Department of Health.

Word count: 2201

Number of references: 19

$\underline{\text { Number of figures: } 6}$

Number of tables: 5 
1 Abstract

2 Purpose To evaluate current practices in anterior chamber (AC) inflammation 3 assessment amongst uveitis specialists.

4 Methods Uveitis specialists were invited to participate in an electronic survey

5 designed to understand their practice in assessing AC inflammation.

6 Results Sixty-five ophthalmologists participated in the survey. Of them, $69.2 \%(\mathrm{n}=$ 7 45) reported using the current Standardization of Uveitis Nomenclature (SUN) 8 guidelines of a 1 x 1-mm slit beam when grading AC cells. Only 38.5\% $(\mathrm{n}=25)$

9 reported routinely counting the number of cells. In the management of uveitis, $98.5 \%$ $10(n=64)$ valued flare assessment, but $84.6 \%(n=55)$ did not use laser flare 11 photometry. In total, $36.9 \%(\mathrm{n}=24)$ agreed that laser flare photometry would change 12 their management, while $16.9 \%(n=11)$ did not see its usefulness. The remaining 13 participants were undecided.

14 Conclusion A number of issues limit the clinical assessment of AC inflammation. 15 Different classifications are still being used despite efforts to standardize practice. 16 While the value of flare is widely recognized, the role of laser flare photometry 17 remains controversial.

18 Keywords Anterior chamber flare; Anterior chamber cells; Anterior chamber activity 19 grading; Laser flare photometry 
28 The breakdown of the blood-aqueous barrier in anterior uveitis results in the release

29 of inflammatory cells and proteins into the aqueous humor. Anterior chamber (AC) 30 activity can manifest as the presence of aqueous cells and flare, leading to the

31 formation of a hypopyon, and also of fibrin in some cases. These features not only

32 assist in the diagnosis of uveitis, but also determine the severity of the disease, 33 providing useful information regarding response to therapy.

35 The assessment of anterior chamber activity is essential to any ophthalmic exam. 36 However, the practice of examining AC activity remains varied among 37 ophthalmologists. The 2 main reasons for this are the use of different systems in 38 grading $\mathrm{AC}$ cells and the continued debate on the usefulness of assessing aqueous 39 flare.

41 Traditionally, AC inflammation is assessed using slit-lamp biomicroscopy. A beam of

42 light is cast posterior to the cornea to examine for any signs of inflammation. For 43 decades, Hogan et al's [1] grading systems were the most widely adopted 44 classifications. Using a 'wide beam and narrow slit' on slit-lamp biomicroscopy, they 45 classified AC activity as shown in Tables 1 and 2.

47 Over time, the system for the grading of AC inflammation was gradually modified. 48 Currently, a number of different grading systems exist [2-5]. These classifications 49 began to specify the size of the slit beam used: $3 \times 1-\mathrm{mm}$ and $2 \times 1-\mathrm{mm}$ slit beams 50 were reportedly being used $[6,7]$. 
52 In 2005, the Standardization of Uveitis Nomenclature (SUN) group was set up with

53 the aim of achieving a consensus on clinical data reporting in the field of uveitis [5].

54 One of the outcomes attained was the agreement in grading of AC cells and flare. A

55 6-step grading system was agreed for the cellular reaction, as documented in Table 3.

56 In comparison with previous systems, there was a minor change in the number of cells

57 that qualified for each grade. The group also agreed on the use of a smaller, $1 \times 1-\mathrm{mm}$

58 slit beam. In the grading of AC flare (Table 4), a 4+ grade was added to the original

59 Hogan et al classifications.

60

61 Many clinicians use cell counts as the benchmark to assess AC inflammation. The usefulness of flare grading, on the contrary, has been questioned. This is because the

63 clinical assessment of flare is qualitative in nature. Some clinicians also believe that

64 flare is an indicator of chronicity rather than of activity [8]. However, it has been 65 argued that cells and flare are both useful markers in grading AC activity because they 66 can both present in varying degrees. The presence of flare may even manifest prior to 67 that of $\mathrm{AC}$ cells in active disease [9].

68

69 Clinically assessing flare is also highly observer-dependent. Previous publications 70 have shown the level of discordance amongst clinicians in grading flare and have 71 highlighted the importance of the clinician's experience level in the accurate grading 72 of flare [11-13]. Additionally, a wide variation of laser flare photometry readings for 73 each step on the clinical scale has been reported, emphasizing the lack of sensitivity 74 when depending solely on the observer's eye $[12,13]$. Therefore, laser flare 
75 photometry is regarded as a more objective method of flare assessment and has also

76 been found to better use the information gathered from flare assessment [10].

The discordance in AC activity assessment not only has significant clinical

79 implications but also is an important aspect to consider when conducting research.

80 This paper aims to identify standard practices and areas of differences in assessing

81 AC activity amongst uveitis specialists from across the world.

\section{Methods}

86 Uveitis specialists from various leading tertiary eye referral centers across the world were invited to participate in the study's survey. Electronic copies of the survey were mailed to the participants. The survey questions posted are shown in Appendix 1.

89 When compared with previous grading systems, the change in the slit beam size 90 specified in the SUN classification was the most significant modification. Hence, 91 question 1 was aimed at determining the different sizes used by the participants. 92 Question 2 examined if it was a common practice to count cells. While this practice is

93 time-consuming and its clinical implications are uncertain, it would still produce the 94 most accurate and consistent grading. Questions 3 to 6 sought to determine 95 participants' perspectives about the usefulness of flare and flare photometry.

97 The Fisher exact test was used to determine the association between responses and the 98 participants' geographical locations. The statistical analysis was performed using R 99 version 3.02 (R Foundation for Statistical Computing, Vienna, Austria). 
101 Approval for the study was obtained from the local ethics review board in accordance 102 with the Declaration of Helsinki.

\section{Results}

107 We received 65 responses out of 180 invitations (36.1\%). Forty of the respondents 108 were from Asia; 15, from Europe including the UK; and 10, from the United States. 109 The results are presented in Figures 1 to 6.

111 A total of $69.2 \%$ of the participants $(n=45)$ reported using the current SUN 112 guidelines to use a $1 \times 1-\mathrm{mm}$ slit beam in their grading of AC inflammation; $7.7 \%(\mathrm{n}=$ 113 5) reported using a 2 x 1-mm slit beam; and $21.5 \%(n=14)$, reported using a $3 \times 1$ $114 \mathrm{~mm}$ slit beam (Fig. 1). One of the participants reported not using the slit lamp for 115 grading AC flare. As the participant thought that the question was in reference to flare 116 grading alone, none of the provided options were selected in the returned response to 117 question 1.

119 A total of $38.5 \%(n=25)$ of the participants reported that they counted the number of 120 cells when grading; $21.5 \%(n=14)$, that they rarely did so; and $4.6 \%(n=3)$, that 121 they never did so (Fig. 2). A significant number of participants $(84.6 \%, \mathrm{n}=55) \mathrm{did}$ 122 not use laser flare photometry in their practice (Fig. 3). However, the majority $123(98.5 \%, \mathrm{n}=64)$ reported seeing the value of flare assessment in the management of 124 uveitis (Fig. 4). Specifically, half of the participants $(49.2 \%, \mathrm{n}=32)$ found flare to be 
of 'very significant' value (Fig. 4), while the majority $(72.3 \%, \mathrm{n}=47)$ agreed that it was a useful marker of disease activity (Fig. 5).

128 When asked if the availability of laser flare photometry would alter their 129 management, we received a mixed response. A total of $36.9 \%(n=24)$ felt that the 130 use of laser flare photometry would be a useful addition to the assessment of their 131 patients and would likely change their management. Only $16.9 \%(n=11)$ did not see 132 any use in laser flare photometry. The remaining participants $(46.2 \%, \mathrm{n}=30)$ were 133 undecided (Fig. 6).

135 Table 5 displays the breakdown of the responses according to the specialists' 136 geographical location. Asia had a lower proportion of participants who always count 137 the number of cells on the slit lamp than did the UK/Europe and the United States $(P$ $138<0.001)$. The UK/Europe had a higher proportion of participants who use laser flare 139 photometry than did Asia and the United States $(P=0.004)$. Asia had a lower 140 proportion of participants who considered flare assessment in uveitis management to 141 be of very significant value than did the UK/Europe and United States $(P=0.001)$. 142 The responses of the 3 groups for the remaining questions did not differ significantly.

\section{Discussion}

147 AC cell grading is an integral part of any ophthalmic examination. However, clinical 148 grading has been marred by interobserver disparity [11]. On the basis of our results, it 
149 is apparent that differences in examination methods are a contributing factor toward 150 this disparity.

152 The SUN classification resulted in the standardization of the slit beam size, which 153 considerably changed the grading of cells as compared with previous classifications 154 [5]. However, our results indicate that a significant number of uveitis specialists do 155 not follow this system, choosing instead to use different-sized slit beams and not to 156 count the number of cells routinely. The specialists from Asia, in particular, appear to 157 count cells less frequently than do their western counterparts (Table 5). The grading 158 of AC cells is such a fundamental activity, one that is so ingrained in a clinician's 159 daily practice, that the need to make any adjustments might not be deemed necessary. 160 For this very reason, some clinicians may not even be aware of the differences in 161 grading systems. This finding is noteworthy because the use of different systems can 162 result in clinically significant interobserver differences.

164 Further questions could also be posed regarding the incongruities in AC inflammation 165 assessment. For instance, the rationale behind the use of a specific slit size was not 166 provided in the SUN publication. To date, the advantage of using a $1 \mathrm{x} 1-\mathrm{mm}$ slit 167 beam over a $3 \times 1-\mathrm{mm}$ slit beam has not been clarified. In all the available grading 168 systems, the ideal location of the slit beam in the AC is similarly not specified. The 169 number of fields a clinician needs to scan in the AC before being able to grade the AC 170 cells accurately is also unclear. Moreover, whether the AC activity should be assessed 171 in a dilated or a nondilated pupil has not been specified in any of the published 172 guidelines. 
174 Besides these differences, the inherent shortcoming of the clinical grading of cells 175 also lies in the fact that it is a nonlinear and semiquantitative system. Although laser 176 cell photometry technology has been described as an objective alternative, the 177 difficultly in differentiating pigments from cells and the technical complexities 178 involved in using this device have limited its usefulness [14].

180 Uveitis is a common disease and is frequently encountered by ophthalmologists from 181 other subspecialties. It is possible that the discrepancies in the assessment of $\mathrm{AC}$ 182 inflammation could be larger between each group of clinicians. While the exact merits 183 of each grading system are unknown, it is more important that a standardized grading 184 system is acknowledged and adhered to. This not only would allow for continuity of 185 care to be preserved in the follow-up of patients but also would maintain the accuracy 186 of data collection in research settings.

188 The majority of the ophthalmologists who participated in our survey agreed that flare 189 is a marker of activity. Participants also generally agreed that flare had a role to play 190 in the diagnosis and management of uveitis. However, most of the participants 191 indicated that they do not use laser flare photometry. This is surprising given that the 192 flaws in the conventional method of assessing AC flare have been widely reported [9, 193 11, 12, 15]. For example, in Kempen et al's [11] study, although interobserver AC 194 flare grading demonstrated a good agreement rate, most cases were graded $0.5+$ to $1+$, 195 even in cases with severe uveitis. This finding suggests a wide range of flare activity 196 between grades $0.5+$ and $1+$ and highlights a possible flaw in the current 197 classification. This finding is supported by that of Agrawal et al [13], who observed a 198 wide range of laser flare readings within each clinical grade of flare. 
200 Laser flare photometry is far superior to the clinical assessment of AC flare on slit-

201 lamp biomicroscopy in terms of precision and reproducibility $[12,13,16]$. It can also 202 play an important role in improving disease management $[13,17,18]$. In fact, Guex203 Crosier et al [19] observed that when the laser flare readings of patients with Behçet's 204 disease were followed up, a 20\% rise in laser flare readings was seen as the earliest 205 sign of recurrence, even before the onset of AC cells. However, our results indicate 206 that many uveitis specialists are still unsure of whether laser flare photometry will 207 change their management practices. As the assessment of flare remains highly valued, 208 further evidence is needed to support the use of laser flare photometry.

210 We believe this study has demonstrated a disparity in the assessment practices of 211 uveitis specialists and hope that it raises questions that will initiate further research 212 and discussion in this area.

214 A limitation of this study is the lack of follow-up questions addressing the reasons for 215 the participants' responses. For instance, it would be informative to find out 216 participants' rationales behind their use of different grading systems. Also, it would 217 be particularly useful to determine why some participants did not use laser flare 218 photometry. For the participants from Asia, the smaller proportion who valued flare 219 assessment could explain the lack of flare photometry use (Table 5). Lack of 220 equipment availability or financial constraints could also be a plausible reason. 221 Follow-up questions would certainly have enabled a better understanding of 222 participants' views towards laser flare photometry. 
224 Given the larger proportion of participants from Asia, the differences found among 225 the different regions may also be skewed and require broader 226 confirmation. Furthermore, whether these differences are specific to individual 227 countries remains uncertain, because we did not analyze the participants by country 228 owing to the small sample size.

230 In summary, the clinical assessment of AC inflammation is a fundamental part of an 231 ophthalmic examination. It is imperative to use a standardized clinical grading scale, 232 especially in the assessment of AC cells. While the current literature recognizes the 233 value of assessing AC flare, its evaluation on the slit lamp is not ideal. Laser flare 234 photometry has long been studied and remains the only objective quantitative method 235 for examining aqueous inflammation. However, no consensus on the use of this 236 method has been reached, and many uveitis specialists still refrain from its use.

237 Further research needs to be conducted to determine the exact role of laser flare 238 photometry in the management of uveitis. 


\section{References}

250 1. Hogan MJ, Kimura SJ, Thygeson P. Signs and symptoms of uveitis: I. Anterior uveitis. Am J

251 Ophthalmol. 1964;47:155-70.

252 2. Schlaegel T. Essentials of uveitis. Boston, MA: Little, Brown, \& Company; 1967.

253 3. Nussenblatt RB, Whitcup SM. Uveitis: fundamentals and clinical practice. 3rd ed. Philadelphia, PA:

254 Mosby; 2004.

255 4. Foster CS, Vitale AT. Diagnosis and treatment of uveitis. Philadelphia, PA: W.B. Saunders

256 Company; 2002.

257 5. Jabs DA, Nussenblatt RB, Rosenbaum JT; Standardization of Uveitis Nomenclature Working Group.

258 Standardization of uveitis nomenclature for reporting clinical data: results of the first international

259 workshop. Am J Ophthalmol. 2005;140:509-16.

260 6. Herbort, Carl P. Appraisal, work-up and diagnosis of anterior uveitis: a practical approach. Middle

261 East Afr J Ophthalmol. 2009;16:159-67.

262 7. Kanski JJ, Bowling B. Clinical ophthalmology: a systemic approach. $7^{\text {th }}$ ed. Philadelphia, USA:

263 Elsevier; 2011.

264 8. Whitcup SM. Examination of the patient with uveitis. In: Nussenblatt RB, Whitcup SM, eds. Uveitis:

265 fundamentals and clinical practice. 4th ed. Philadelphia, USA: Mosby; 2010.

266 9. Ladas JG, Wheeler NC, Morhun PJ, Rimmer SO, Holland GN. Laser flare-cell photometry:

267 methodology and clinical applications. Surv Ophthalmol. 2005;50:27-47.

268 10. Sawa M, Tsurimaki Y, Tsuru T, Shimizu H. New quantitative method to determine protein

269 concentration and cell number in aqueous in vivo. Jpn J Ophthalmol. 1988;32:132-42.

270 11. Kempen JH, Ganesh SK, Sangwan VS, Rathinam SR. Interobserver agreement in grading activity

271 and site of inflammation in eyes of patients with uveitis. Am J Ophthalmol. 2008;146:813-8.

272 12. Konstantopoulou K, Del'omo R, Morley AM, Karagiannis D, Bunce C, Pavesio C. A comparative

273 study between clinical grading of anterior chamber flare and flare reading using the Kowa laser flare

274 meter. Int Ophthalmol. 2012;35:629-33.

275 13. Agrawal R, Keane PA, Singh J, Saihan J, Kontos A, Pavesio CE. Comparative analysis of anterior 276 chamber flare grading between clinicians with different levels of experience and semi-automated laser 277 flare photometry. Ocul Immunol Inflamm. 2014;26:1-10.

278 14. Li Y, Lowder C, Zhang X, Huang D. Anterior chamber cell grading by optical coherence 
tomography. Invest Ophthalmol Vis Sci. 2013;54:258-65.

280 15. Tugal-Tutkun I, Herbort CP. Laser flare photometry: a noninvasive, objective, and quantitative

281 method to measure intraocular inflammation. Int Ophthalmol. 2010;30:453-64.

282 16. Bernasconi O, Papadia M, Herbort CP. Sensitivity of laser flare photometry compared to slit-lamp

283 cell evaluation in monitoring anterior chamber inflammation in uveitis. Int Ophthalmol. 2010;30:495-

284500.

285 17. Fang W, Zhou H, Yang P, Huang X, Wang L, Kijlstra A. Longitudinal quantification of aqueous

286 flare and cells in Vogt-Koyanagi-Harada disease. Br J Ophthalmol. 2008;92:182-5.

287 18. Fang W, Zhou H, Yang P, Huang X, Wang L, Kijlstra A. Aqueous flare and cells in Fuchs 288 syndrome. Eye. 2009;23:79-84.

289 19. Guex-Crosier Y, Pittet N, Herbort CP. Sensitivity of laser flare photometry to monitor

290 inflammation in uveitis of the posterior segment. Ophthalmology. 1995;102:613-6. 
Figure Legends

Fig. 1 Size of slit beam used

Fig. 2 Frequency of counting the number of anterior chamber cells

Fig. 3 Percentage of respondents using laser flare photometry

Fig. 4 Significance of flare in management of uveitis

Fig. 5 Percentage of respondents who consider flare a useful marker of disease activity

Fig. 6 Percentage of respondents who think laser flare photometry can change uveitis management 